\section{Comunicação em saúde: evidências e desafios na prevenção do VIH em mulheres trabalhadoras de sexo na cidade de Maputo, Moçambique}

Health communication: evidence and challenges in HIV prevention among female sex workers in Maputo city, Mozambique

Maltez Alberto Mabuie
RELIGACIÓN

REVISTA DE CIENCIAS SOCIALES Y HUMANIDADES JOURNAL OF SOCIAL SCIENCES AND HUMANITIES REVISTA DE CENCIAS SOCIAIS E HUMANAS

INFORMACIÓN

http://doi.org/10.46652/rgn.v6i30.863 ISSN $2477-9083$

Vol. 6 No. $30,2021$. e210863 Quito, Ecuador

Enviado: octubre 18, 2021

Aceptado: diciembre 12, 2021

Publicado: diciembre 18, 2021

Publicación Continua

Sección Sur-Sur| Peer Reviewed álcool, prática de sexo desprotegido e desconhecimento do estado serológico por parte das MTS. As ações de comunicação em saúde que são dirigidas por educadoras de pares privilegiam apenas a disseminação de informação. É um fato o fraco envolvimento das MTS na concepção de ações de comunicação em saúde e na segmentação de audiência. Conclui-se que, até hoje não se quis envolver e conhecer as caraterísticas e necessidades das MTS, providenciar sessões que vão para além da disseminação de informação, estruturar programas de mobilização comunitária assentes em redes sociais de convivência, assim como romper as barreiras estruturais que concorrem para a propagação do VIH nos locais de concentração das MTS.

Palavras-chave: Informação deficitária; mudança de comportamento; sexo desprotegido; VIH

\begin{abstract}
Health communication is becoming increasingly relevant in the design and implementation of programmes that aim to contribute to the prevention and treatment of diseases in the population. This research aims to understand the health communication actions in the prevention of human immunodeficiency virus (HIV) among female sex workers (FSW) in Maputo city. A case study of qualitative nature was conducted with a sample of $150 \mathrm{FSW}$ selected through key informants. The results indicate that sex work tends to start early, there is alcohol abuse, unprotected sex and lack of knowledge of HIV status by FSW. Health communication actions by peer educators gives priority only to the dissemination of information. It is a well-known fact that there is poor involvement of FSW in the design of health communication actions and in audience segmentation. The conclusion is that, to date, there has been no attempt to involve and learn about the characteristics and needs of the FSW to provide sessions that go beyond the dissemination of information, to structure community mobilization programs based on social networks of coexistence, and to break down the structural barriers that contribute to the spread of HIV in places where FSW are concentrated.
\end{abstract}

Keywords: behaviour change; HIV; inadequate information; unprotected sex; HIV
Conflicto de interesses

O autor declara que não há possível conflito de interesses.

Financiamiento

Não houve assistência financeira de partes externas a este artigo.

Agradecimiento

Associação Bedjany Vavassate va Moçambique - ABEVAMO.

Nota

O presente artigo é um recorte de uma pesquisa de Mestrado em Jornalismo e Estudos Editoriais e tinha como objetivo compreender as ações de comunicação em saúde na prevenção do VIH nas MTS na cidade de Maputo.

ENTIDAD EDITORA 


\section{Introdução}

A comunicação faz parte da rotina diária do homem, permitindo a interação através da troca de mensagens, valores e sentimentos. Campos (2015), defende que existe uma tendência de confundir informação com comunicação, chegando-se até a atribuir o mesmo significado. Todavia, informar não é comunicar; a informação só se torna comunicação quando está agregada a um relacionamento, à interação, ao processo pelo qual os interlocutores precisam de estar em sintonia.

Na área da saúde, a comunicação tem um papel essencial na influência de comportamentos, uma vez que as doenças não deveriam ser vistas como sendo resultado de um fator biológico único, mas também como resultado de causas múltiplas.

A comunicação em saúde tem uma função essencial na concepção e implementação de ações que contribuam para a prevenção e o tratamento de doenças na população. Tais ações beneficiam a população em geral, mas principalmente grupos mais vulneráveis num país como Moçambique que vive uma epidemia generalizada do vírus da imunodeficiência humana (VIH) (CNCS, 2015a).

Dentre os grupos mais vulneráveis, as Mulheres Trabalhadoras de Sexo (MTS) estão mais expostas. O Inquérito Integrado Biológico e Comportamental (IBBS) entre MTS indica uma prevalência do VIH significativamente maior que a prevalência entre a população feminina geral, de mesma faixa etária, 15-49 anos. Em Maputo, a prevalência do VIH entre MTS é de 31,2\%, e 16,8\% na população em geral e 50\% das seropositivas desconhecia a sua condição de portadora do vírus (INS, et al., 2013).

O quarto plano estratégico nacional de resposta ao VIH e SIDA 2016-2020 (PEN IV) considera as MTS como um grupo com prioridade para intervenções de prevenção do VIH, em Moçambique e principalmente na cidade de Maputo (CNCS, 2015a).

Os locais de concentração das MTS, onde a vulnerabilidade econômica, as redes sexuais extensas, e os altos níveis de abuso de álcool, drogas e de violência criam um ambiente de risco elevado. As organizações não governamentais (ONG), organizações de base comunitária (OBC) e o governo moçambicano, são responsáveis por ações de comunicação em saúde, principalmente nos locais de maior concentração das MTS, no intuito de reforçar a resposta ao VIH.

A comunicação está associada à intervenção social na área da saúde, podendo ser responsável pelas vidas da população, por isso um interesse maior nos dias atuais (Moraes, 2008). Existem evidências relevantes de que os programas de comunicação em saúde, quando bem organizados e executados, podem influenciar comportamentos, na busca de um ambiente favorável para a provisão de serviços de saúde (JHU, 2009).

Segundo Dasgupta (2021), a prevenção do VIH pode ser alcançada através da promoção da participação em ações de comunicação em saúde voltadas às vivências e realidades de marginalização das MTS. Dessa forma, o presente estudo tem o objetivo de compreender as ações de comunicação em saúde na prevenção do VIH nas MTS na cidade de Maputo.

A compreensão de tais ações é de extrema importância, de forma a validá-los. Neste contexto, o desenho de estratégias voltadas à dinâmica dos locais de concentração das MTS, podem fornecer elementos para uma melhor concepção e implementação de ações de comunicação em saúde. 


\section{Metodologia}

Foi conduzido de julho a dezembro de 2019, um estudo de caso, com natureza qualitativa, de caráter descritivo numa amostra de 150 MTS. A amostra foi selecionada por dez informanteschave em 15 locais de concentração das MTS na rua de Bagamoyo, cidade de Maputo (Distrito Urbano de KaMubukwana). As informantes-chave são MTS com função de líderes, dirigindo sessões de educação de pares. Foi adotada a amostragem não probabilística e cada informantechave recrutou dez MTS.

A coleta de dados ocorreu por observação e pela aplicação de questionários, formulados de forma a tratar dos aspetos associados aos objetivos estabelecidos. As participantes foram informadas sobre o estudo, convidadas a participar do mesmo, e foi garantida a confidencialidade dos dados obtidos; cada participante assinou o termo de consentimento livre e esclarecido. As MTS com menos de 15 anos e as não autodeclaradas como MTS foram excluídas, bem como as que não estavam em atividade nos seis meses anteriores à aplicação do questionário. Não houve compensação financeira pela participação.

\section{Resultados e discussão}

A rua de Bagamoyo na cidade de Maputo é um dos maiores centros de venda de sexo em Moçambique e as MTS oferecem os seus serviços para visitantes, residentes e trabalhadores de instituições públicas e privadas nos arredores.

Na Tabela 1 está representado o perfil obtido a partir das observações e questionários administrados.

Tabela 1. Perfil das MTS

\begin{tabular}{|lcc|}
\hline \multicolumn{3}{|c|}{ n (150) } \\
15 e 18 anos & Faixa etária \\
19 a 24 anos & 62 & $41,3 \%$ \\
25 a 39 anos & 41 & $27,3 \%$ \\
40 a 49 anos & 36 & $24 \%$ \\
mais de 49 anos & 5 & $3,3 \%$ \\
& 6 & $4 \%$ \\
nível primário & Escolaridade & \\
sem formação & 67 & $44,7 \%$ \\
nível básico & 38 & $25,3 \%$ \\
nível médio & 32 & $21,3 \%$ \\
& 13 & $8,6 \%$ \\
até um ano & Tempo de trabalho & \\
mais de 3 anos & 72 & $48 \%$ \\
mais de 4 anos & 66 & $44 \%$ \\
\hline
\end{tabular}

Fonte: preparado pelo autor.

O perfil das MTS é composto por mulheres com a faixa etária entre 15 e 18 anos (41,3\%), seguido da faixa etária de 19 a 24 anos (27,3\%) e 25 a 39 anos (24\%), uma pequena percentagem pertence 
à faixa etária entre 40 e 49 anos (3,3\%) e com mais de 49 anos (4\%). Os resultados encontrados corroboram o início precoce do trabalho de sexo e estão de acordo com os dados encontrados em Maputo, Beira e Nampula (INS, et al., 2013).

Diversos organismos internacionais da área da saúde, definem MTS como sendo mulheres, acima dos 18 anos de idade, que trocam serviços sexuais por dinheiro ou bens regular ou ocasionalmente (MISAU, 2016). Na realidade, o presente estudo comprovou que parte significativa das mulheres que fazem trabalho de sexo, encontram-se abaixo dos 18 anos. Tal informação é alarmante e obriga a uma reflexão profunda frente às questões ligadas à saúde e justiça.

Quanto à escolaridade, foi verificado que a maioria das MTS possui o nível primário (44,7\%), seguido de $25,3 \%$ sem formação, 21,3\% com nível básico e apenas 8,6\% possui nível médio de educação. Tais dados indicam o abandono da escola por parte das adolescentes. Algumas participantes relataram a existência de universitárias entre elas, a despeito destas não se exporem a seus pares ou conhecidos, optando, talvez, pela prestação de serviços ao domicílio.

Os dados obtidos demonstram que $48 \%$ das MTS estão no trabalho de sexo há um ano, 44\% há mais de três anos e $8 \%$ há mais de quatro anos. Os dados denotam que diversas mulheres estão a aderir ao trabalho de sexo na rua de Bagamoyo. O aumento considerável de mulheres no trabalho de sexo poderá, de alguma forma, demandar do governo um maior investimento em programas de prevenção e tratamento do VIH e SIDA. Ao considerar que a epidemia do VIH é generalizada, bem como o fato de que uma parte considerável das MTS está em relações estáveis, e que os seus parceiros poderão ter outras parceiras, haverá um efeito em cascata da infeção.

Em relação ao ambiente, tanto comportamental como estrutural à disposição das MTS, o trabalho de sexo ocorre, na grande maioria dos casos, nos estacionamentos e áreas livres, com anuência e cobrança por parte dos guardas. As MTS apontaram que quando saem da rua, existe um risco elevado de não receberem pelo serviço prestado, serem agredidas ou não terem acesso ao uso de preservativo. O trabalho de sexo ainda acontece dentro dos carros dos clientes e hotéis, mas com menor incidência.

Tabela 2. Ambiente Comportamental e Estrutural observado

\begin{tabular}{|lcc|}
\hline & n (150) & \% \\
consumo de álcool & & $75,3 \%$ \\
regularmente & 113 & $8 \%$ \\
raramente & 12 & $6,7 \%$ \\
ocasionalmente & 10 & $5,3 \%$ \\
sempre & 8 & $4,7 \%$ \\
Nunca & 7 & $50,3 \%$ \\
uso do preservativo & & $26 \%$ \\
sempre & 83 & $18,6 \%$ \\
regularmente & 39 & $34,7 \%$ \\
ocasionalmente & 28 & $65,3 \%$ \\
sexo desprotegido por violência & & \\
Sim & 52 & $23,3 \%$ \\
Não & 98 & $20,7 \%$ \\
sexo sem preservativo por um valor maior & & $56 \%$ \\
ocasionalmente & 35 & $64,7 \%$ \\
raramente & 31 & $35,3 \%$ \\
nunca & 84 & \\
Testagem ao VIH & & \\
nunca fez o teste & 97 & 53 \\
já fez o teste & &
\end{tabular}

Fonte: preparado pelo autor. 
Em relação ao estilo de vida das MTS na rua de Bagamoyo, a venda de bebidas alcoólicas na via pública, bares e discotecas favorece o consumo por elas. De acordo com os dados, 75,3\% delas relataram consumir regularmente álcool enquanto exercem o trabalho; $8 \%$ raramente; $6,7 \%$ ocasionalmente; 5,3\% consumiam sempre e 4,7\% relataram nunca consumir álcool. O consumo aumenta enormemente a vulnerabilidade e especialmente diminui a perceção de risco de infeção em relação ao VIH, bem como outras doenças ou agravos à saúde.

O não uso ou uso incorreto do preservativo é um dos maiores riscos aos quais as MTS estão expostas, muitas vezes de forma propositada por parte de clientes (INS, et al., 2013). Locais específicos para a atividade poderiam reduzir tal risco, já que durante a pesquisa as mulheres foram vistas em locais sem iluminação ou sem um mínimo de conforto, o que poderia facilitar o rompimento do preservativo e aumento da transmissão de doenças sexualmente transmissíveis.

As MTS declaram que são assediadas, constantemente, a fazer sexo sem preservativo, chegando a receber pagamentos maiores ao fazê-lo, parte delas $(34,7 \%)$ também relata relações sexuais desprotegidas como resultado de agressões infligidas pelos clientes, alguns dos quais são representantes da polícia.

Segundo a Aids Fonds (2016), não existe uma relação harmoniosa entre os agentes legais e as MTS, muitos deles abusam do seu poder em troca de dinheiro ou sexo, fato este revoltante quando se considera que tais profissionais são, constantemente, alvo de treinamentos sobre direitos humanos e proteção de populações vulneráveis. Em Moçambique, este grupo encontra várias barreiras para aceder aos diversos serviços fundamentais, agravando o seu estado de vulnerabilidade (MISAU, 2016). As MTS da pesquisa também acentuaram o estigma e a discriminação que enfrentam em virtude do seu trabalho incluindo familiares, membros da comunidade e colegas. Destes grupos, as participantes descreveram abusos verbais e físicos e, em alguns casos até, relatos de estupro coletivo. Tais episódios de estigmatização podem manter as MTS apartadas dos serviços dedicados à saúde e de assistência médica.

Tabela 3. Envolvimento das MTS em exercícios de segmentação de audiência e ações de comunicação

\begin{tabular}{|c|c|c|}
\hline & n (150) & $\%$ \\
\hline \multicolumn{3}{|c|}{ Participação em exercícios de segmentação de audiência } \\
\hline Não & 150 & $100 \%$ \\
\hline Sim & o & $0 \%$ \\
\hline \multicolumn{3}{|c|}{$\begin{array}{l}\text { envolvimento no desenho de ações de comunicação para a prevenção do } \\
\text { VIH }\end{array}$} \\
\hline Não & 150 & $100 \%$ \\
\hline Sim & o & $0 \%$ \\
\hline
\end{tabular}

Fonte: preparado pelo autor.

As MTS participantes do estudo afirmaram não terem participado do processo de mapeamento e $100 \%$ relataram não ter participado de exercícios de segmentação de audiência, que levam em conta a idade e o período em atividade. A segmentação de audiência permite selecionar os públicos-alvo com base nos fatores de risco para a saúde, comunicação efetiva de mensagens de saúde complexas e identificação de canais e estratégias de comunicação (Karanesheva, 2015). 
Considera-se que a segmentação das MTS é influenciada pelo comportamento no risco de infeção pelo VIH (CNCS, 2015b). É fato que a relação sexual é um assunto privado do comportamento humano e a segmentação de audiência poderia ser integrada na abordagem programática, auxiliando MTS a se prevenirem de novas infeções.

Assim, como as MTS nunca participaram de sessões de segmentação de audiência, as ações de comunicação na rua de Bagamoyo são conduzidas sem planificação, sem saber da necessidade de cada grupo. A ideia defendida por Bandura et al. (2008), percursor da teoria social cognitiva, que considera que as pessoas aprendem novos comportamentos observando as ações de outros, os programas de comunicação em saúde na rua de Bagamoyo deveriam ter uma estrutura organizada e maior coordenação entre os vários atores envolvidos. Não seria possível conhecer o comportamento de cada uma das MTS sem que os programas de comunicação em saúde fossem sistematizados.

O National Institute of Cancer (2004), defende que a comunicação em saúde só pode produzir mudanças sustentadas, quando acompanhada por regulamentos e políticas a favor do públicoalvo. Tal afirmação, quando extrapolada à realidade das MTS, traz uma triste realidade: as MTS afirmaram que na rua de Bagamoyo existem ações de comunicação visando a prevenção do VIH, desenvolvidas por ONG e OCB, através de sessões de educação de pares, teatro e disponibilização de material de informação, educação e comunicação, no entanto 100\% das que participaram na pesquisa relatam não terem sido envolvidas no desenho de ações de comunicação e mensagens para a prevenção do VIH nos últimos 24 meses.

Os pesquisadores de Palo Alto admitem que o recetor é tão importante quanto o emissor, dentro do processo comunicativo (Wolf, 2003). Ainda nesta perspetiva de responsabilidade, Campos (2015), considera que a intenção comunicativa é a consciência e compreensão de que um indivíduo pode influenciar pessoas por meio da comunicação, modificar o seu ambiente e atingir resultados. $\mathrm{Na}$ rua de Bagamoyo, as ações de comunicação desenvolvidas pelas EP não são capazes de alimentar a cadeia do processo comunicativo, principalmente na área da mudança de comportamento.

Em relação à prevenção do VIH, as participantes expressaram a necessidade de ter mais informações sobre a saúde, e tal demanda é comprovada quando avaliados os resultados que mostram que somente $50,3 \%$ das entrevistadas utilizam preservativo sempre, $26 \%$ usam regularmente e $18,6 \%$ ocasionalmente. O número de MTS que ocasionalmente utilizam o preservativo é alarmante, e mostra a necessidade de ações estruturadas de comunicação em saúde para que se conheça as características do grupo e suas convicções, de forma a modificar o seu comportamento. Além deste grupo, as MTS mantêm relações sexuais sem uso do preservativo quando o cliente promete pagar um valor superior ao habitual, nesta questão $23,3 \%$ das entrevistadas admitiu que o faz ocasionalmente, $20,7 \%$, raramente e $56 \%$ relatou nunca ter aceite.

Dentro do longo e árduo caminho que as ações de comunicação necessitam trilhar encontra-se o conhecimento do seu estado serológico, primeiro passo para responder à problemática do VIH e SIDA. A comunicação em saúde deve ser entendida de forma integral, considerando o sujeito na sua totalidade, as suas dificuldades, os seus anseios, e não meramente como o repasse de informações rebuscadas com o uso de termos técnicos (Ribeiro et al., 2013). 


\section{Conclusão}

O presente estudo teve como propósito compreender as ações de comunicação em saúde e a sua eficácia na prevenção da infeção pelo VIH nas MTS num dos maiores centros de venda de sexo em Moçambique.

De acordo com os resultados encontrados, a faixa etária mais predominante das MTS é dos 15 aos 18 anos, mostrando o início precoce do trabalho de sexo, tal característica concorre com o nível de escolaridade baixo ou inexistente. Os dados também evidenciam que muitas mulheres estão a aderir ao trabalho de sexo na rua de Bagamoyo, com base no número de mulheres (48\%) trabalhando há menos de um ano.

O ambiente estrutural e comportamental apresenta vários desafios, uma vez que o trabalho de sexo acontece em espaços que possibilitam violência física e sexual e, muitas vezes, limitam o acesso ao uso do preservativo.

O consumo de álcool é preocupante, deixando as MTS mais vulneráveis e sem capacidade de discernimento, deixando-as vulneráveis a propostas de relações sexuais desprotegidas, na promessa de um pagamento maior que o habitual. Há relatos, por parte das MTS, de ações de comunicação em saúde que privilegiam a disseminação de informação impulsionadas por EP, provenientes de ONG e OBC, porém na ausência de uma estrutura funcional das redes de MTS no local. Os dados indicam ainda o fraco envolvimento das MTS na concepção de ações de comunicação e no desenho de mensagens para a prevenção do VIH, além de não ser realizada a segmentação de audiência. Em relação ao objetivo proposto, ressaltamos que:

1. As ações de comunicação em saúde, necessitam de um ambiente estrutural e comportamental mais preservado (o que parece não ser o caso, levando em consideração a falta de segurança e proteção, o consumo excessivo de álcool, a localização dos pontos onde ocorre o trabalho de sexo e a falta do uso do preservativo).

2. As ações de comunicação em saúde levadas a cabo na cidade de Maputo não privilegiam a segmentação da audiência.

3. Há falta de envolvimento das MTS no desenho de ações de comunicação em saúde.

Para potenciar as ações de comunicação em saúde, é necessário envolver e conhecer as caraterísticas e necessidades das MTS, incluir na planificação o uso de teorias da comunicação e modelos de mudança de comportamento como é o caso da Escola de Palo Alto e a Teoria Social Cognitiva, providenciar sessões que extrapolem a disseminação de informação, estruturando programas de mobilização comunitária condizentes com as redes sociais de convivência, assim como romper as barreiras estruturais que concorrem para a propagação do VIH nos locais de concentração das MTS.

Isto posto, os resultados frisam a necessidade de uma maior coordenação por parte dos comunicadores para a prevenção do VIH, junto às MTS na rua de Bagamoyo, já que esta é, e provavelmente será por um longo período de tempo, a maior preocupação das autoridades da saúde em relação à epidemia do VIH. É de destacar que tal prevenção, embora focada, não está restrita, uma vez que tanto as MTS quanto os clientes mantêm relações com outros parceiros e, assim, facilmente o vírus se propaga. Igualmente, a prevenção poderá atingir uma parcela cada vez maior da população. 


\section{Referências}

Aids Fonds. (2016). Trabalho de sexo e violência em Moçambique. Relatório de avaliação de necessidades. Aids Fonds.

Bandura, A., Azzi, R.G., \& Polydoro, S. (2008). Teoria social cognitiva: Conceitos básicos. Artmed.

Campos, L. (2015). Comunicação. Laureate International Universities.

CNCS. (2015a). Plano Estratégico Nacional de Resposta ao HIV e SIDA (2016-2020). CNCS.

CNCS. (2015b). Padrões nacionais para educação de pares \& alcance para a prevenção e cuidados do HIV no seio da população chave. CNCS.

Dasgupta, S. (2021). Community-Based Strategies as Transformative Approaches for Health Promotion and Empowerment among Commercial Sex Workers in India. Sexes, 2, 202-215. https://doi.org/10.3390/ sexes2020018

INS, CDC, UCSF, Pathfinder International \& I-TECH. (2013). Relatório final: Inquérito integrado, biológico e comportamental entre mulheres trabalhadoras de sexo. UCSF.

JHU. (2009). Summary report: reviewing existing evidence on the contribution of communication interventions to increasing family planning use. JHU.

Karanesheva, T. (2015). Role of the audience segmentation for the health communication process. Bulgarian Journal of Public Health, 7, 2, 61-73.

MISAU. (2016). Directriz para integração dos serviços de prevenção, cuidados e tratamento em HIV e SIDA para a população chave no sector da saúde. MISAU

Moraes, A. (2008). Informação estratégica para as ações de intervenção social na saúde. Ciência \& Saúde Coletiva, 13, 2, 2041-2048.

National Cancer Institute. (2004). Making health communication programs work. Estados Unidos da América. National Cancer Institute.

Ribeiro, C., Cruz, A., \& Maríngolo, A. (2013). Comunicação em Saúde: Conceitos e estratégias, rumo à efetivação de direitos sociais [Apresentação de pôster]. III Simpósio Mineiro de Assistentes Sociais, Belo Horizonte, Brasil.

Wolf, M. (2003). Teorias da comunicação. Presença.

\section{Autor}

Maltez Alberto Mabuie. Docente pesquisador na Escola Superior de Jornalismo em Moçambique. Atua principalmente nas seguintes áreas: informação em saúde, necessidades e comportamento informacional, biblioteconomia social, comunicação para a mudança social e de comportamento, comunicação de riscos em emergências de saúde pública e direitos humanos. 